\title{
Multiscale Modeling of Intergranular Fracture in Aluminum: Constitutive Relation For Interface Debonding
}

\author{
V. Yamakov ${ }^{1 *}$, E. Saether ${ }^{2}$, and E.H. Glaessgen ${ }^{2}$ \\ ${ }^{1}$ National Institute of Aerospace, Hampton, VA 23666, USA \\ ${ }^{2}$ NASA Langley Research Center, Hampton, VA 23681, USA
}

Intergranular fracture is a dominant mode of failure in ultrafine grained materials. In the present study, the atomistic mechanisms of grain-boundary debonding during intergranular fracture in aluminum are modeled using a coupled molecular dynamics - finite element simulation. Using a statistical mechanics approach, a cohesive-zone law in the form of a traction-displacement constitutive relationship, characterizing the load transfer across the plane of a growing edge crack, is extracted from atomistic simulations and then recast in a form suitable for inclusion within a continuum finite element model. The cohesive-zone law derived by the presented technique is free of finite size effects and is statistically representative for describing the interfacial debonding of a grain boundary (GB) interface examined at atomic length scales. By incorporating the cohesive-zone law in cohesive-zone finite elements, the debonding of a GB interface can be simulated in a coupled continuum-atomistic model, in which a crack starts in the continuum environment, smoothly penetrates the continuum-atomistic interface, and continues its propagation in the atomistic environment. This study is a step towards relating atomistically derived decohesion laws to macroscopic predictions of fracture and constructing multiscale models for nanocrystalline and ultrafine grained materials.

\footnotetext{
*Corresponding author: Tel.: +757-864-2850; fax: +757-864-8912.

E-mail address: yamakov@nianet.org (V. Yamakov)
} 


\section{Introduction}

Physics-based modeling of fracture begins at nanometer dimensional scales in which atomistic simulation is used to predict the evolution of various deformation mechanisms on a fundamental level. These mechanisms include dislocation nucleation and interaction, interstitial void formation, and atomic diffusion. The development of these damage mechanisms progresses into microscale processes such as local plasticity and small crack formation. Ultimately, damage progression leads to macroscopic failure modes such as plastic yielding and large cracks exhibiting Mode I, II, and III behavior. To capture this macroscopic behavior, the use of direct atomistic simulations is impractical due to their strong inherent limitations in size and time scales. Thus, multiscale modeling strategies are needed for the purpose of minimizing computational requirements and developing a unified description of the hierarchy of material processes that govern fracture.

Recently, a multiscale strategy using a constitutive relation-based continuum modeling of the process of intergranular fracture in aluminum has been proposed [1,2]. In this strategy, information from the lower atomistic level is transferred to the upper continuum level through constitutive relations governing different damage modes. The constitutive relations must reflect the most dominant characteristics of the individual deformation mechanisms revealed at the lower atomistic level to adequately model the behavior observed at higher length scales. For fracture simulations, the traction-displacement relationship (as introduced by Dugdale and Barenblatt $[3,4])$, is used to model the cohesive properties of the grain boundary (GB) interface for a specific fracture mode. The traction-displacement relationship in this strategy is extracted from atomistic molecular dynamics (MD) simulations [5], and is incorporated into cohesive-zone models (CZM) [6] used in finite element models [7]. This yields a finite element analysis 
capability to study the behavior of metallic microstructural damage at the grain scale $[1,2]$. In this multiscale approach, the CZM elements represent the mechanical response of the GBs as obtained from atomistic simulations, while the constitutive properties of the crystalline grains are represented by continuum finite elements with appropriate anisotropic elastic properties. Thus, an integrated multiscale modeling strategy for understanding the connection between macroscopic fracture and the underlying atomistic failure mechanisms emerges. In principle, the range of relevant length scales achieved by this strategy can span from the atomistic $\left(10^{-9} \mathrm{~m}\right)$ to the macroscopic $\left(>10^{-3} \mathrm{~m}\right)$.

An essential part of this strategy is the extraction of CZMs for interface decohesion from an atomistic simulation. Efforts on this topic have been made by various groups in the last few years [8-11]. All of those simulations showed a highly elevated stress of debonding ranging from 15 to $50 \mathrm{GPa}$, which are generally two orders of magnitude higher than the experimentally observed strength of the corresponding material [8]. There are various reasons for this discrepancy. For example, the approach used in all of these works [8-11] was based on simulating the debonding of a flat interface under a constant tensile strain rate perpendicular to the interface. The dynamics of the atoms was severely constrained by the boundary conditions, which did not allow for Poisson contraction and prevented shear deformation. As a result, plastic processes, such as dislocation slip, interface sliding and interface diffusion, were strongly suppressed. Consequently, the simulated mechanism for interface decohesion in these works reproduced an idealized process of atomic adhesion (strength) rather than fracture at the interface.

Recently, a methodology for extracting CZMs from atomistic simulations of crack propagation has been developed [5]. The main goal of this methodology was to extract and understand the contributions of different atomistic processes to an MD-based CZM decohesion 
law for intergranular fracture under local conditions of a propagating crack. A new concept of defining cohesive-zone-volume elements (CZVE) as atomic subdomains encompassing the debonding interface, and presenting an atomistic equivalent of CZM elements was introduced. In this concept, the CZM is viewed as a statistical representation of a large, Gibbs-type ensemble of CZVEs placed along the crack path during crack propagation. The CZM is obtained as a statistical average of the traction-displacement states of the CZVE ensemble under the conditions of a steady-state crack propagation.

In this paper, the concept of implementing a statistical CZVE ensemble to extract a CZM constitutive law in terms of a traction-displacement relationship will be used in an improved coupled atomistic-continuum model for intergranular fracture in aluminum. The dependence of the loading conditions and temperature on the CZM will be determined. The derived CZM will be incorporated within finite elements introduced in the continuum domain of the coupled atomistic-continuum model as an extension of the atomistic interface. This provides the ability to simulate crack growth smoothly propagating from the continuum region into the atomistic domain. This technique allows multiscale atomistic-continuum modeling of fracture processes where fracture may not be confined to the atomistic domain.

\section{The simulation approach}

The simulation approach used in this study is based on a coupled atomistic-continuum model that allows a large atomistic domain (containing $10^{6}$ or more atoms) to be embedded within a continuum domain of micron dimensions. The atomistic domain is simulated using MD, where the interatomic forces are represented by an atomistic potential suitably fitted to reproduce the material properties of aluminum [12]. The continuum domain is simulated by using the finite 
element method (FEM) with anisotropic elastic properties derived from the aluminum potential [12] at room temperature $(300 \mathrm{~K})$. The coupling between FEM and MD is achieved by the recently-developed embedded statistical coupling method (ESCM) $[13,14]$ to provide elastic boundary conditions for the atomistic domain and transfer the applied far field mechanical load into the atomistic system.

The ESCM method is based on the concept that the continuum representation of a material, in terms of stress-strain fields, is a statistical representation of its atomic structure. Following this idea, the connection between the atomistic and the continuum representations at the $\mathrm{MD} / \mathrm{FE}$ interface is performed using a statistical mechanics approach. The method uses statistical averaging over both time and volume of atomistic subdomains at the $\mathrm{MD} / \mathrm{FE}$ interface to provide nodal displacement boundary conditions to the continuum FEM domain. The FEM generates interface reaction forces that are uniformly distributed over the interface atoms in the form of constant traction boundary conditions [15] to the MD domain. Thus, this approach is based on solving the special boundary value problem (BVP) at the MD/FE interface for a coupled MDFEM system and may be described as a local-nonlocal BVP because it relates local continuum nodal quantities with statistical averages of nonlocal atomistic quantities over selected atomic volumes. For this study, one finite element at the interface encompasses a region of several hundred to several thousand atoms, which positions have been averaged over 1 ps time interval. At those scales, the discreteness of the atomic structure is sufficiently homogenized so that the FEM domain responds to the atomistic domain as an extension of the continuum. The constant traction boundary conditions of the MD domain ensure that the elastic field from the FEM domain is correctly transferred to the atomistic region. An iterative procedure using the MD statistical displacements establishes a balance between the FEM-computed forces and the MD- 
computed forces at the interface. This force balance ensures stress-displacement continuity at the interface.

The model used to extract CZMs for a given grain boundary in aluminum is presented in Fig. 1. A circular MD domain with a diameter $d_{\mathrm{MD}}=90 \mathrm{~nm}$ was embedded in a square FEM mesh with side dimension $d_{\mathrm{FE}}=20 \mathrm{~d}_{\mathrm{MD}}$. In this example, the system represents a bicrystal with a $<11$ $0>\sum 99$ symmetric tilt GB passing along the line $(y=0)$ in Fig. 1. In the MD domain, the grain boundary was formed by symmetrically rotating the two crystals at $89.42^{\circ}$ with respect to each other around a common tilt axis coinciding with the $\left[\begin{array}{lll}1 & 1 & 0\end{array}\right]$ crystallographic direction, chosen as the $z$-direction in Fig. 1 . The system thickness in the $z$ - direction was $h=2.9 \mathrm{~nm}$ and includes 10(1 110$)$ crystallographic stacking planes. Periodic boundary conditions were applied in the $z$ direction of the MD system to emulate a bulk atomic state in this direction. Structurally, the atomistic model is equivalent to the one discussed in detail in [5]. The MD stress along the periodic $z$-direction in the MD domain was maintained at zero by applying the ParrinelloRahman constant stress technique [16]. The MD simulation was performed at constant temperature using the Nose-Hoover thermostat [17].

\section{Atomistic derivation of CZM}

To derive the CZM for interface debonding, an edge crack along the GB line (Fig. 1), which extended from one of the outer boundaries of the FEM mesh to the interface with the MD domain, was inserted in the coupled MD-FEM model. The crack was propagated into the MD domain by imposing a tensile uniaxial strain of $1.5 \%$ in the $y$-direction (perpendicular to the crack plane). The atomistic processes associated with crack propagation along this GB were studied in detail in [5]. Energetic analysis of the different dissipative mechanisms during crack 
growth was given in [18], and some aspects of the dynamics of the fracture process were reported in [19].

CZVEs of size $l \times 2 l \times h$ in the $x$-, $y$ - and $z$-directions, respectively, with $l=1.9 \mathrm{~nm}$, were introduced along the GB plane as discussed in [5] (Fig. 2). Each CZVE contained approximately 1250 atoms over which the stress-displacement response of the element was calculated. During crack propagation, the data from each CZVE was used to extract profiles of the normal stress, $\sigma_{y y}(x)$, and opening-displacement, $\lambda(x)$, along the GB interface. Figure 2 shows an example of one of these profiles, together with a snapshot of the atomistic configuration, from which it was extracted. A fit, using $\sigma_{y y}=K_{I} / \sqrt{2 \pi\left(x-x_{0}\right)}$, gave an estimate of the stress intensity factor of $K_{\mathrm{I}}$ $=0.88\left[\mathrm{MPa}-\mathrm{m}^{1 / 2}\right]$ for $x_{0}$ defined at the position of the crack tip. This value is slightly lower than the stress-intensity factor for a pure linear elastic model of the same geometry, $K_{\mathrm{I}}=1.09[\mathrm{MPa}-$ $\mathrm{m}^{1 / 2}$ ], estimated from [20]. The discrepancy is most likely a result of the developed plasticity at the crack tip in the atomistic simulation.

Using a statistical mechanics approach [21], the CZM is derived in the following way. The state of each CZVE at position $x$ and instant of time $t$ is defined by the state variables $\sigma_{y y}(x, t)$ and $\lambda(x, t)$. The CZVE state is represented as a point $\left(\lambda, \sigma_{y y}\right)$ in a $\sigma_{\mathrm{yy}}-\mathrm{Vs}-\lambda$ configuration space. In the statistical limit of steady-state crack propagation that occurs over an infinitely long time over an infinitely long interface, all realized CZVE states would produce a $\rho\left(\lambda, \sigma_{y y}\right)$ distribution (density of states) that is a continuous function independent of time. The CZM is defined as a statistical average, $\bar{\sigma}_{y y}(\lambda)$, of $\sigma_{y y}$ over a small interval $(\lambda-\Delta \lambda, \lambda+\Delta \lambda)$ of this distribution 


$$
\bar{\sigma}_{y y}(\lambda)=\left\langle\sigma_{y y}(\lambda)\right\rangle=\frac{\int_{\lambda^{\prime}=\lambda-\Delta \lambda \sigma_{y}^{\prime}=-\infty}^{\lambda+\Delta \lambda} \int_{\lambda^{\prime}=\lambda-\Delta \lambda}^{\infty+\lambda \lambda} \int_{\sigma_{y y}^{\prime}=-\infty}^{\infty} \rho\left(\lambda^{\prime}, \sigma_{y y}^{\prime}\right) d \sigma_{y y}^{\prime} d \lambda^{\prime}}{\int_{y y}^{\prime}}
$$

at the limit of $\Delta \lambda \rightarrow 0$.

Numerically, $\rho\left(\lambda, \sigma_{y y}\right)$ can be approximated by using the already described coupled MDFEM simulation model. For a finite number of measurements, $\rho\left(\lambda, \sigma_{y y}\right)$ takes a discrete form

$$
\rho\left(\lambda, \sigma_{y y}\right)=\sum_{i} \delta\left(\lambda-\lambda_{i}\right) \delta\left(\sigma_{y y}-\sigma_{y y}\left(\lambda_{i}\right)\right),
$$

where the sum is over all measured data points $\left(\lambda_{i}, \sigma_{y y}\left(\lambda_{i}\right)\right)$ and $\delta\left(x-x_{i}\right)$ is the Dirac delta function.

After substituting $\rho\left(\lambda, \sigma_{y y}\right)$ from Eq. (2) into Eq. (1), $\bar{\sigma}_{y y}(\lambda)$ takes the form of a moving average

$$
\bar{\sigma}_{y y}(\lambda)=\left\langle\sigma_{y y}(\lambda)\right\rangle=\frac{1}{N\left(\lambda_{i} \in\{\lambda-\Delta \lambda, \lambda+\Delta \lambda\}\right)} \sum_{i=1}^{N} \sigma_{y y}\left(\lambda_{i}\right)
$$

where the sum is taken over all $N$ points $\left(\lambda, \sigma_{y y}\right)$, which are between $\lambda-\Delta \lambda$ and $\lambda+\Delta \lambda$. The choice of the size of the interval, $\Delta \lambda$, depends on the desired resolution of the CZM with respect to $\lambda$.

Figure 3 shows the derived $\rho\left(\lambda, \sigma_{y y}\right)$ for the example given in Fig. 2 (the case for $K_{\mathrm{I}}^{(1)}=1.09$ $\left[\mathrm{MPa}-\mathrm{m}^{1 / 2}\right]$ ), after collecting data from 180 snapshots that were taken at intervals of $1 \mathrm{ps}$. Another example with a lower stress intensity of $K_{\mathrm{I}}^{(2)}=0.72\left[\mathrm{MPa}-\mathrm{m}^{1 / 2}\right]$ with all other conditions being equal, is also given for comparison. The solid lines, drawn through both distributions in Fig. 3, are the averaged $\bar{\sigma}_{y y}(\lambda)$ CZM curves (Eq. 3). Both CZMs were derived using a resolution of $\Delta \lambda=0.1 \mathrm{~nm}$. 
The comparison between the two CZMs in Fig. 3 indicates that increasing the stress intensity in the simulation increases the displacement at full debonding, $\lambda_{0}{ }^{(1)}>\lambda_{0}{ }^{(2)}$, while the peak stress, $\sigma_{\mathrm{p}}=6.4 \mathrm{GPa}$, is approximately constant. Here, the increase in $K_{\mathrm{I}}$, is associated with an increase in plastic mechanisms (i.e., increased nucleation of dislocations) in the softening region rather than an increase in the force needed to break atomic bonds.

A substantial effect of temperature can be seen on the CZM result at $\mathrm{T}=100 \mathrm{~K}$ and $K_{\mathrm{I}}=0.72$ [MPa-m ${ }^{1 / 2}$ ] shown in Fig. 3 when compared to the CZM result at T=300K shown in Fig. 4 for the same loading conditions. Increasing the temperature reduced the peak stress from $6.3 \mathrm{GPa}$ to 5.4 $\mathrm{GPa}$, but increased the displacement at full debonding from $1.7 \mathrm{~nm}$ to $2.7 \mathrm{~nm}$. The implication is that the work, $\Gamma=\int \sigma_{y y}(\lambda) d \lambda$ (Fig. 4), done in the softening region increases due to increased crack tip plasticity. The decrease in $\sigma_{\mathrm{p}}$ can be explained by the decrease of force required for bond breaking due to the increased atomic thermal motion at higher temperature.

\section{Crack propagation from a continuum to atomistic region}

The next logical step after extracting the CZM curve from atomistic simulations is to implement it within a CZM element and verify that the debonding predicted by the atomistic simulation is reproduced by the finite element analysis. For this purpose, the same MD-FEM coupled model as described in Section 2 was used with a slight, but essential, modification: the atomistic GB in the MD domain was extended into the continuum domain by placing CZM elements along the interface between the two grains. The thickness of the system was the same as that used for extracting CZMs, $h=2.9 \mathrm{~nm}$, while the lateral dimensions were reduced by a factor of two to save simulation time such that $d_{\mathrm{MD}}=45 \mathrm{~nm}$ and $d_{\mathrm{FE}}=900 \mathrm{~nm}$. It was verified that decreasing of the system size did not affect the extracted CZM. 
Again, an edge crack was inserted along the GB line, but unlike the model in Section 3, the crack did not extend all the way to the MD domain. Rather, it had to propagate through four $\mathrm{CZM}$ elements $(12 \mathrm{~nm})$ in the continuum before reaching the $\mathrm{MD} / \mathrm{FE}$ interface. Its growth into the continuum domain is governed by the cohesive zone model incorporated within the CZM. For computational efficiency, this CZM law was defined as a least-square bilinear fit to the extracted $\rho\left(\lambda, \sigma_{y y}\right)$ distribution at $\mathrm{T}=300 \mathrm{~K}$, shown in Fig. 4. The two points, $\left(\lambda_{p}, \sigma_{p}\right)$ and $\left(\lambda_{0}, 0\right)$ were used as fitting parameters (Fig. 5). The so-called "penalty stiffness", defined by the slope of the CZM curve before the maximum traction is reached, was set to match the initial slope (from $\sigma=0$ to $\sigma=\sigma_{\mathrm{p}}$ ) of the atomistic traction-displacement relationship. In this way, the stiffness of the CZM before opening matched the elastic stiffness of the GB interface.

A uniform tensile strain that gradually increased from 0 to $1.5 \%$ was applied to the outer boundaries of the FEM domain. The strain was applied in $0.1 \%$ increments over $10 \mathrm{ps}$ MD time intervals (equivalent to $1 \times 10^{8} \mathrm{~s}^{-1}$ strain rate). This constant strain rate allowed a gradual growth of the edge crack, first in the continuum domain, and then penetrating into the MD domain. The process is shown in several sequential snapshots in Figs. 6(a-f) that focus on the MD domain in the center of the coupled MD-FEM model. In Figs 6(a) and 6(b), the crack propagates through the FEM mesh, sequentially opening the CZM elements placed along its path. In Fig. 6(c), the crack tip enters the MD domain. The snapshot depicts the decohesion at the MD/FE interface in unison with the opening of the last CZM element. In Figs. 6(d) and 6(e), the crack continues its growth in the MD domain. Plastic processes start to develop in the form of dislocation nucleations, seen as short green lines marking the stacking faults at the extended dislocation cores on both sides of the crack tip. Fig. 6(f) presents a crack that has propagated well inside the MD domain. Active dislocation nucleation accompanies the propagation. The snapshots in Figs 
6(a-f) also quantitatively show that both the crack opening and the stress-displacement field are continuous at the $\mathrm{MD} / \mathrm{FE}$ interface during the entire process of crack propagation.

\section{Summary}

This study examined a methodology for the atomistic derivation of a cohesive-zone law that can be implemented in cohesive-zone finite element models for simulating fracture in nanocrystalline or ultrafine grained aluminum. The cohesive-zone law is derived as a statistical mechanics representation of crack propagation through defining an ensemble of atomistic cohesive-zone-volume-elements. The simulation methodology implements a coupled MD-FEM model utilizing statistical coupling between the atomistic and the continuum representations of the material. The model allows large atomistic domains (containing $10^{6}$ or more atoms) simulated by MD at finite temperature to be embedded into an FEM mesh of micrometer dimensions. The cohesive-zone laws derived by this technique are free of finite size effects and are statistically representative for describing the interfacial debonding of an idealized interface examined at atomic length scales. As a test example, a coupled continuum-atomistic model was presented, in which a crack starts in the continuum environment, smoothly penetrates the continuum-atomistic interface, and continues its propagation in the atomistic environment. The model introduces the possibility of conducting multiscale simulations where fracture is not strictly limited to the atomistic region, which may greatly benefit the development of multiscale atomistic-continuum models of nanocrystalline and ultrafine grained materials.

\section{Acknowledgements}


Contributed paper proceeding to the 2008 TMS meeting, New Orleans, LA, March 9-13, 2008.

V. Yamakov is sponsored through cooperative agreement NCC-1-02043 with the National Institute of Aerospace. 


\section{References:}

1. V. YAMAKOV, D. R. PHILliPS, E. SAETHER, and E. H. GLAESSGEN, "Chapter 8. Multiscale Modeling," in Applied Computational Materials Modeling: Theory, Experiment, and Simulation, Bozzolo, Noebe and Abel, eds., Springer, (2007) ISBN 978-0-387-23117-4.

2. E. GLAESSGEN, E. SAETHER, D. PHILliPS, and V. YAMAKOV, $47^{\text {th }}$ AIAA/ASME/ASCE/AHS/ASC Structures, Structural Dynamics and Materials Conference and Exhibit, Newport, RI, May 1-4, 2006.

3. D. S. DUGDALE, J. Mech. Phys. Solids, 8 (1960) 100.

4. G. I. BARENBLATT, Adv. Appl. Mech., 7 (1962) 55.

5. V. YAMAKOV, E. SAETHER, D. R. PHILliPS, and E. H. GLAESSGEN, J. Mech. Phys. Solids 54 (2006) 1899.

6. V. TVERGAARD and J.W. HUTCHINSON, Int. J. Solids Struct., 33, (1996), 3297.

7. G. BEER, Int. J. Numer. Meth. Engrg., 21, (1985), 585.

8. K. GALL, M. F. HORSTEMEYER, M. VAN SCHILFGAARDE, and M. I. BASKES, J. Mech. Phys. Solids, 48 (2000) 2183.

9. R. KOMANDURI, N. CHANDRASEKARAN, L. M. RAFF, Int. J. Mech. Sci., 43 (2001) 2237.

10. D. SPEAROT, K. I. JACOB, and D. L. MCDOWELL, Mech. Mater., 36 (2004) pp. 825.

11. J. E. RAYNOLDS, J. R. SMITH, G. -L. ZHAO, and D. J. SROLOVITZ, Phys. Rev. B, 53 (1996) 13883.

12. Y. MISHIN, D. FARKAS, M. J. MEHL, and D. A. PAPACONSTANTOPOULOS, Phys. Rev. B, 59 (1999) 3393.

13. E. SAETHER, V. YAMAKOV, and E. GLAESSGEN, $49^{\text {th }}$ AIAA/ASME/ASCE/AHS/ASC Structures, Structural Dynamics and Materials Conference, (2007) .

14. E. SAETHER, V. YAMAKOV, and E. GLAESSGEN, (in preparation)

15. F. CLERI, Physical Review B, 65 (2001) 014107. 
16. M. PARRINELLO, and A. RAHMAN, Journal of Applied Physics, 52 (1981) 7182.

17. S. NOSE, Journal of Chemical Physics, 81 (1984) 511.

18. V. YAMAKOV, E. SAETHER, D. R. PHILLIPS, and E. H. GLAESSGEN, J. Mat. Sci., 42 (2007) 1466.

19. V. YAMAKOV, E. SAETHER, D. R. PHILlIPS, and E. H. GLAESSGEN, Phys. Rev. Lettrs., 95 (2005) 015502.

20. Y. MURAKAMI editor, Stress Intensity Factors Handbook, vol. 1., Pergamon Press, New York, 1988.

21. Y. L. KLIMONTOVICH, Statistical Theory of Open Systems, vol. 1, Kluwer Academic Publishers, Netherlands, 1995. 


\section{Figures:}

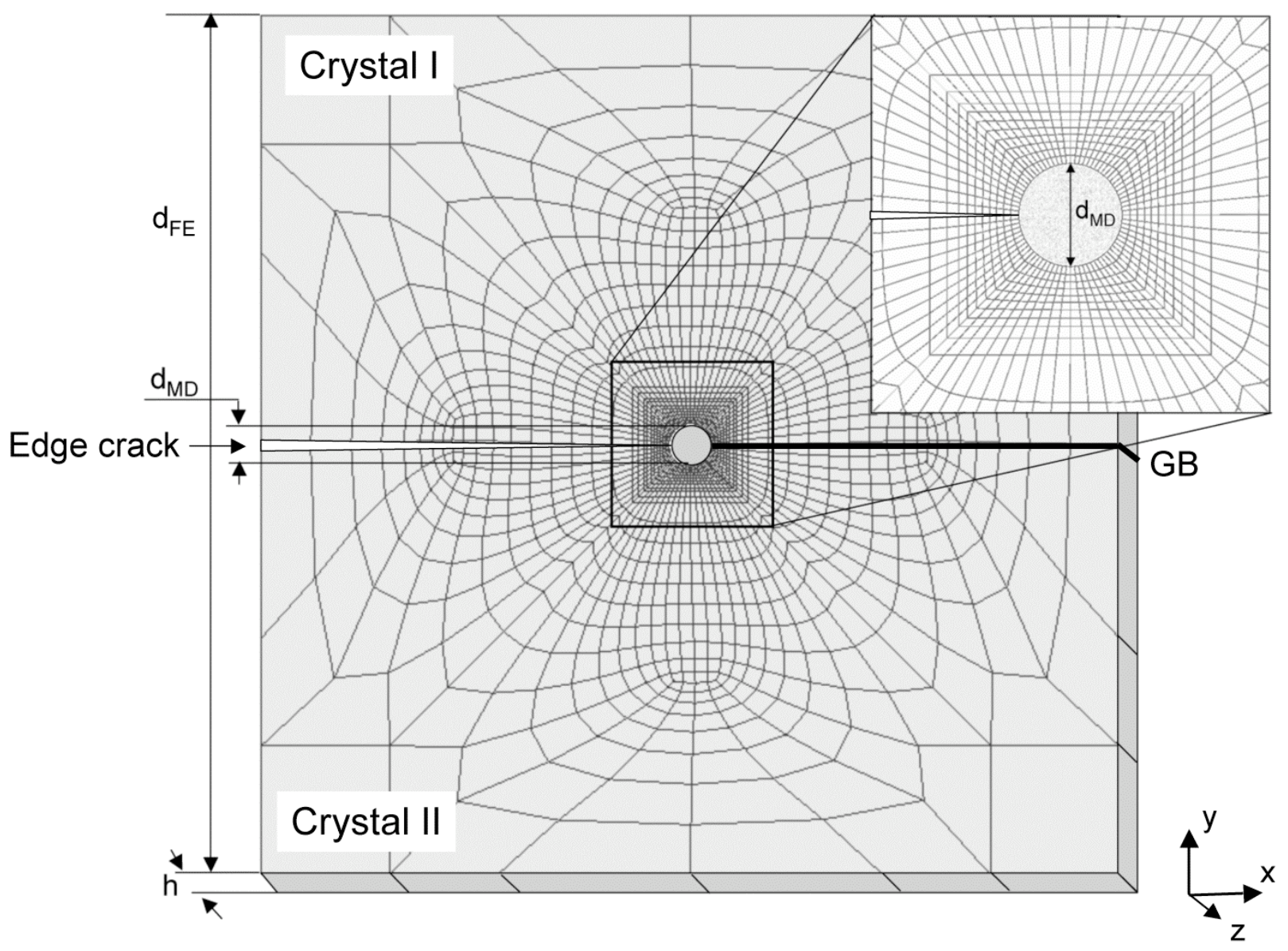

Fig. 1. Schematic description of the ESCM coupled molecular-dynamics - finite-element model configuration. 


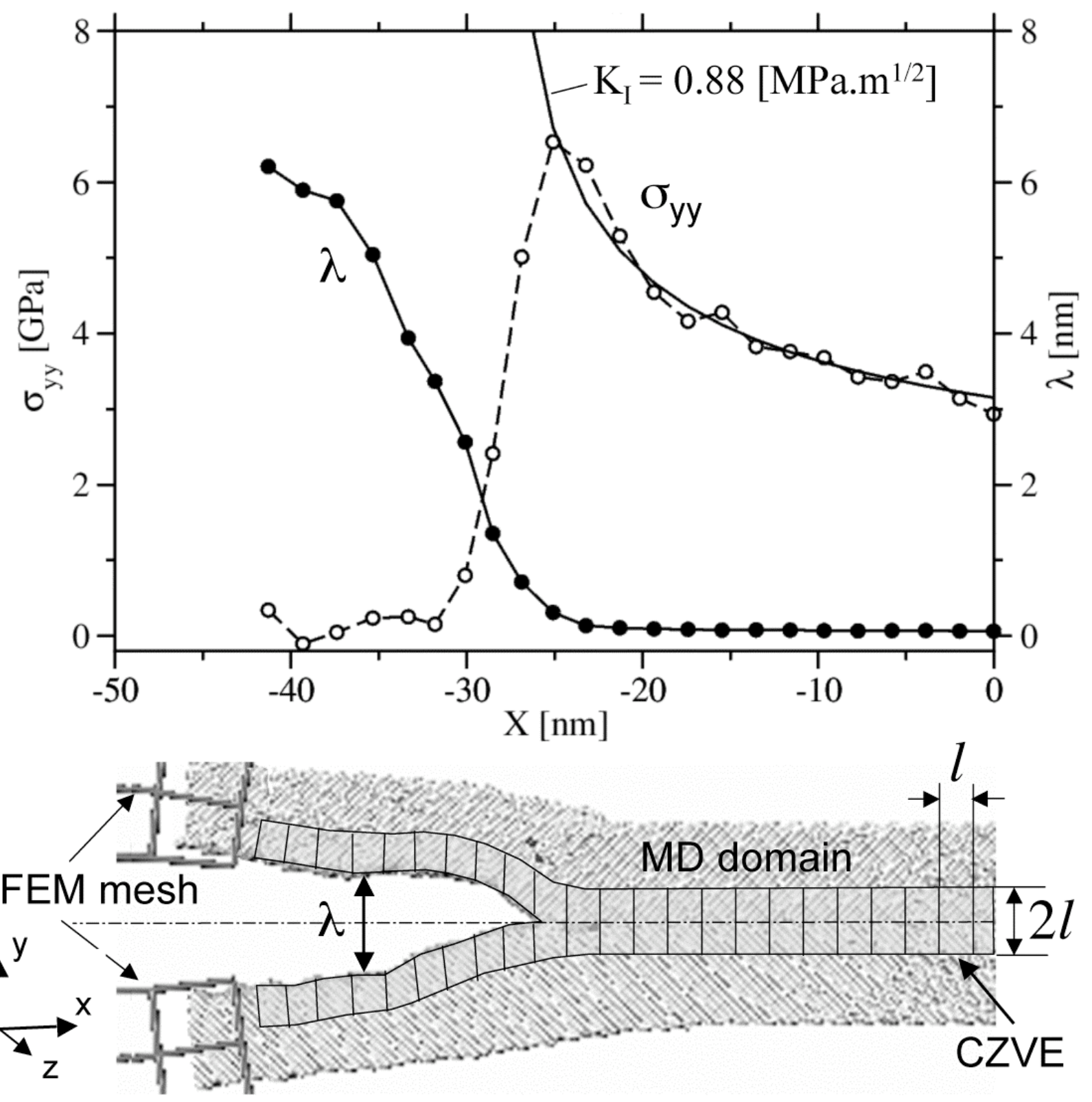

Fig. 2. Stress and opening profiles from cohesive-zone-volume-elements (CZVE) along a grainboundary edge crack growing inside the molecular-dynamics region. 


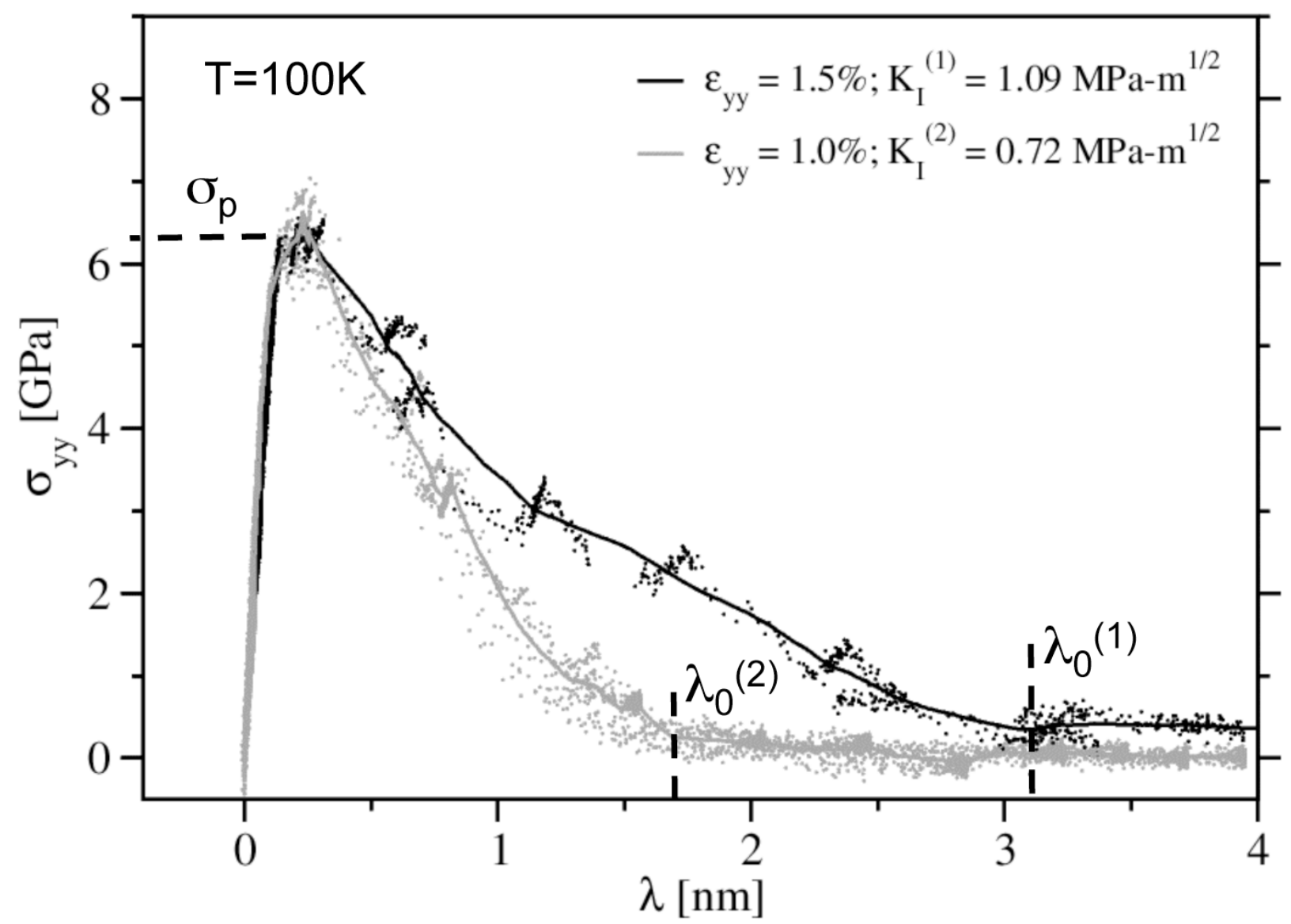

Fig. 3. Statistical distributions of measurements from individual CZVEs (dots), and averaged traction-displacement relationships (solid lines) at $\mathrm{T}=100 \mathrm{~K}$ extracted from the $\mathrm{ESCM}$ model at different stress intensity factors. The values $\sigma_{\mathrm{p}}, \lambda_{0}{ }^{(1)}$, and $\lambda_{0}{ }^{(2)}$, indicate the peak stress, and the displacements at full debonding at $\mathrm{K}_{\mathrm{I}}^{(1)}$ and $\mathrm{K}_{\mathrm{I}}^{(2)}$, respectively. 


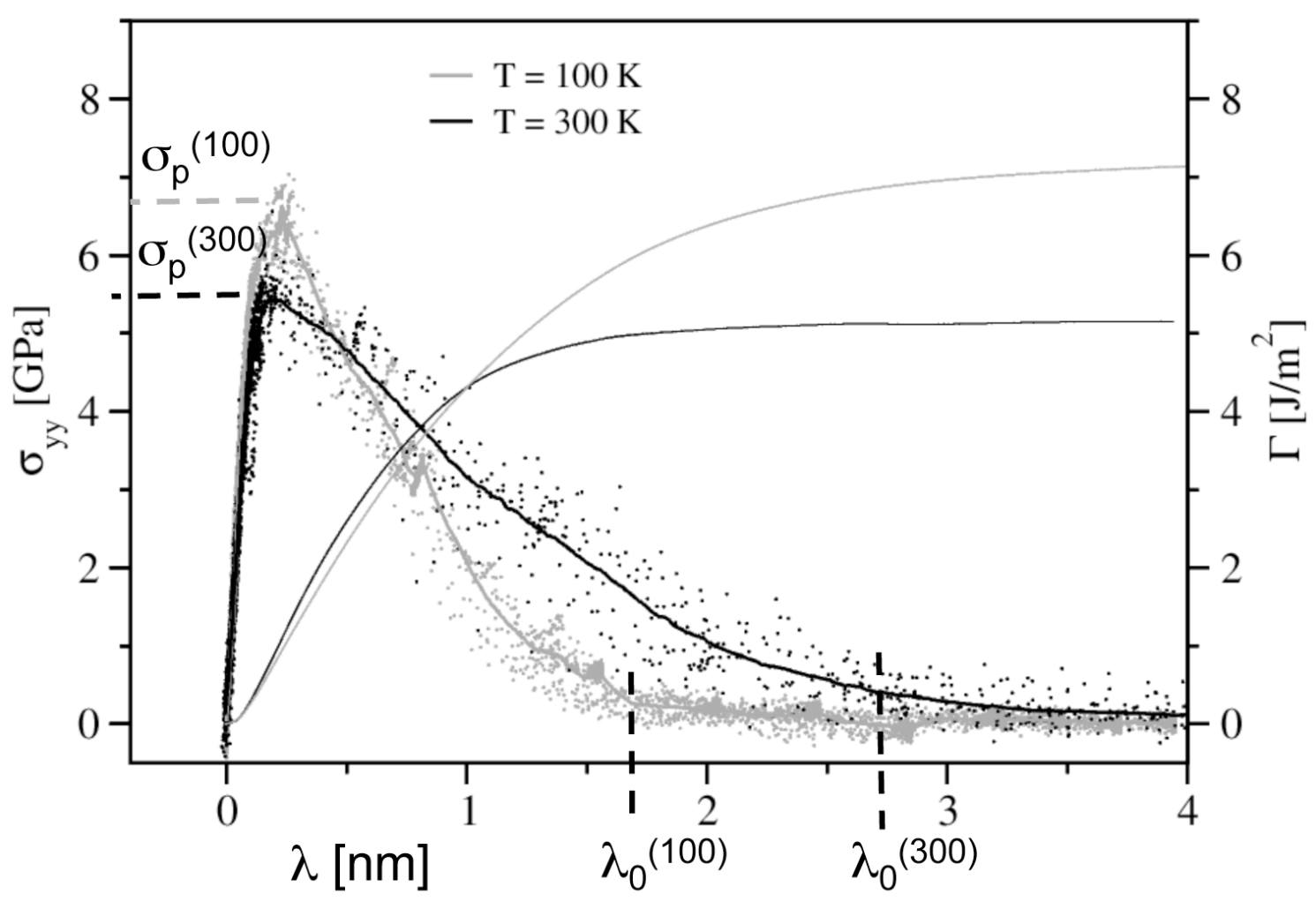

Fig. 4. Traction-displacement relationships $\sigma_{y y}(\lambda)$, and energy of decohesion $\Gamma(\lambda)$, (the solid lines) at $\mathrm{T}=100$ and $300 \mathrm{~K}$. The statistical distributions of measurements from individual CZVEs, used to extract the traction-displacement relationships are also given (dots) for comparison. The values $\sigma_{\mathrm{p}}{ }^{(100)}, \lambda_{0}{ }^{(100)}, \sigma_{\mathrm{p}}{ }^{(300)}$, and $\lambda_{0}{ }^{(300)}$, indicate the peak stress and the displacement at full debonding at $\mathrm{T}=100 \mathrm{~K}$ and $300 \mathrm{~K}$, respectively. 


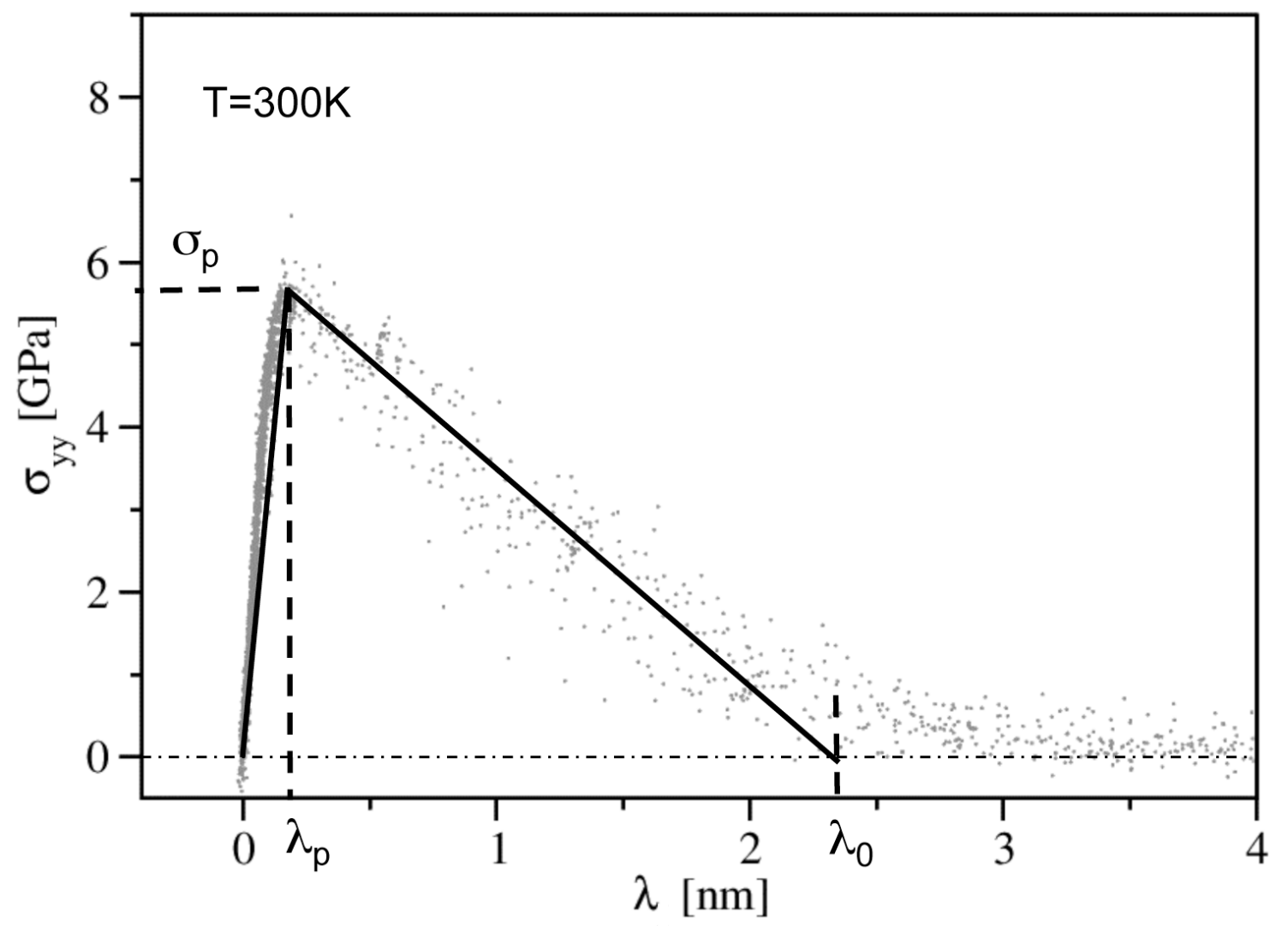

Fig. 5. Bilinear CZM, parameterized as a least-square fit to the extracted statistical stressdisplacement distribution of measurements from individual CZVEs at $\mathrm{T}=300 \mathrm{~K}$. The values $\sigma_{\mathrm{p}}$, $\lambda_{\mathrm{p}}$, and $\lambda_{0}$ are used as parameters. 

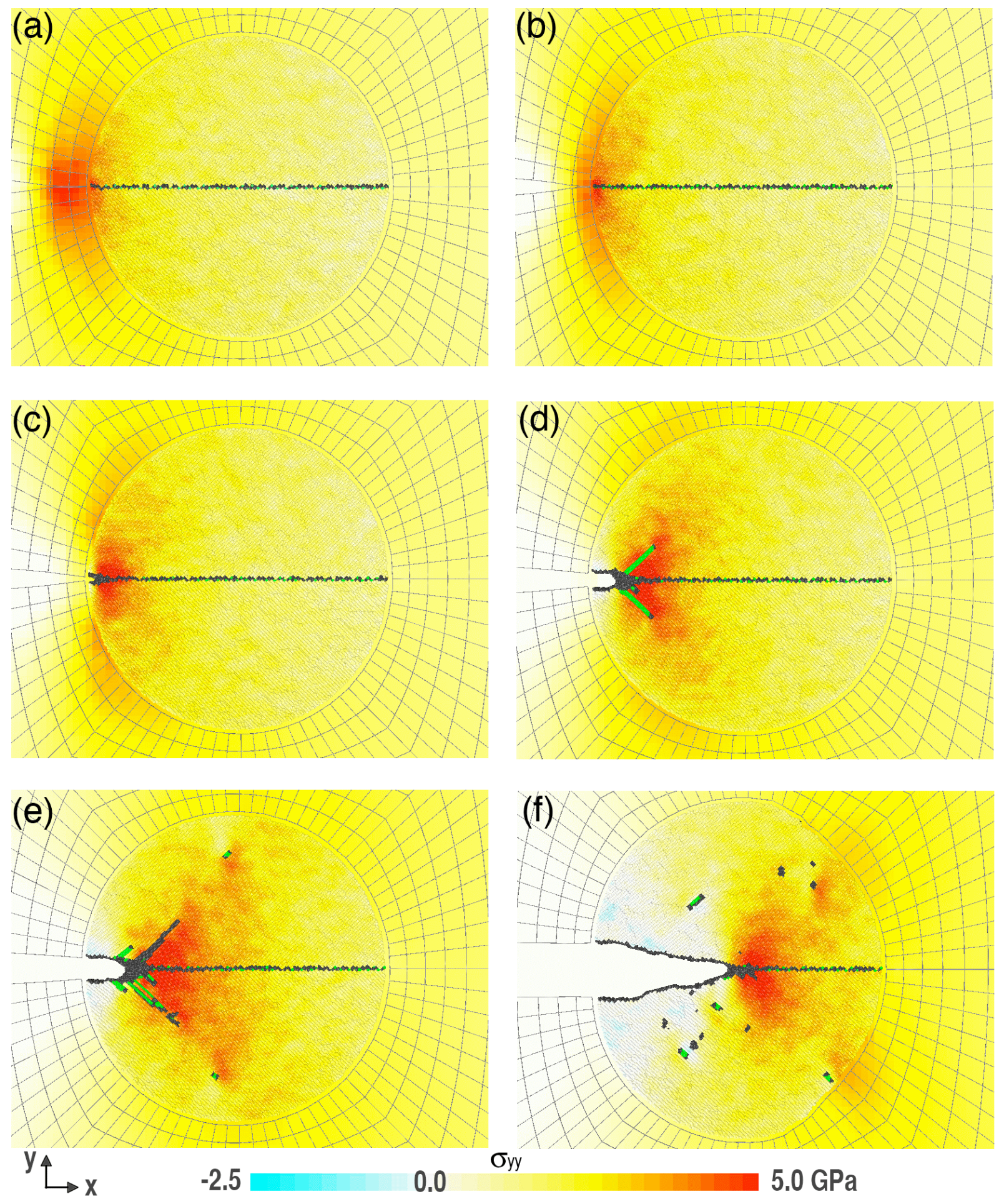

Fig. 6. Structural snapshots with imposed $\sigma_{\mathrm{yy}}$ stress map on the f.c.c atoms of the coupled MDFEM/CZM model simulating an edge crack passing through the MD-FEM interface and debonding a GB in aluminum. Strain rate of $1 \times 10^{8} \mathrm{~s}^{-1}$ was applied. Atoms in h.c.p state (indicating stacking faults produced from partial dislocations) are colored in green, and the rest (disordered and surface atoms) are shown in black. The six snapshots are taken at uniaxial tensile strain $\varepsilon_{y y}$ as follows: (a) $1.2 \%$; (b) $1.3 \%$; (c) $1.3 \%$; (d) $1.4 \%$; (e) $1.5 \%$; (f) $1.5 \%$. 